\title{
Contents, Vol. 116, 1948
}

\section{Index.}

Amsler, M., Das kranke Kammerwasser im Film

199

Diskussion: A. Bruckner, Verrey. Babel, J., L'évolution histologique des pseudo-tumeurs maculaires séniles . 277

Discussion: Dufour.

Babel, J., vide Blum, J. D.

Balavoine, C, vide Motsier, G. de.

Bangerter, A., Aus der operativen Praxis

Diskussion: Goldmann, A. Bruckner, Huber, Waatdenburg.

Bárány, E.H., The Influence of Gum Arabic and Dextran on the Blood-

Aqueous Barrier and Intra-Ocular Pressure 65

Bernoulli, R., Lidkrebs bei einem Hund, ausgehend von der Tarsaldrüse und einem Papillom 101

Bischler, V., La triplopie binoculaire 254

Blum, J. D., et J. Babel, Diagnostic différentiel des reunites et pseudorétinites pigmenlaires au point de vue histologique ...... 261

Discussion: Bietti, Babel, Bornschein, H, Bedeutung und Vermeidung spannungsbedingter Lichtschwan-

kungen bei Adaptometern

Botos, B., and /. Vönõczky, Appearance of Sulfonamides in the Eye .

Bourquin, J, B, Effets thérapeutiques d'un nouveau collyre sulfamidé (solufontamide) .246

Brand, E, Ein neues Schnellverfahren zur Darstellung von Trachomeinschluß-

Körperchen 61

Bruckner, R, Über pharmakologische Beeinflussung des Augendruckes bei verschiedenen Körperlagen 200

Diskussion: Goldmann, Schlußwort: R. Bruckner.

Bürki, E, Weitere Ergebnisse zur Ke > ratoplastik mit Paraffinmaterial . .221

Busacca, A., Quelques maladies cornéennes observées à la lumière de la thérapeutique pénicillinique 43

- Lesions rétiniennes produites par coup de foudre ou par eblouissement? 141 Cometta, F, Sur la conservation du Greffon cornéen dans la paraffine liquide 307 Csillag, F, Entfernung des subluxierten Stares mit verflüssigter Rinde mittels

besonderer Schlinge .

Dellaporta, A., Über Falten in der Retina nach druckherabsetzenden Operationen bei Glaukom 51

- Über Spätinfektion nach den Operationen von Elliot und Lagrange nebst Bemerkungen über die Häufigkeit der Spätinfektion nach Iridencleisis antiglaukomatosa 322 
Dufour, R, Manifestations oculaires dans la tbc. primo-secondaire. Iridocyclite «séreuse» et périphlébite rétinienne $2 \Theta 8$

Fanta, H., Über die Wundsprengung nach Cataractoperationen .

Fischer, F. P., Über die Frequenzkurven der Altersveränderungen des Auges .

Franceschetti, A., Operation à Гæil sympathisant d'une cataracte due à une ophtalmie sympathique .

Francois, J., Kystes des procès ciliaires, observes par la gonioscopie après

enclavement de Гiris. (Avec planche II) ..... . 313

Frischer, M., W. Jablonski und M. Loebel, Über die Behandlung äußerer aller-

gischer Augenerkrankungen in Palãstina ...... 335

Girardet, M., Verres de contact et aphakie ....... 226

Discussion: Blum, Bürki, Dufour. Goldmcnn, H., Über Abflußdruck und Glasstabphänomen. Zur

Palhogenese

des einfachen Glaukoms

Gut, A., Ophthalmoskopie im aufrechten Bild in indirekter Beleuchtung. (Hierzu

Tafel I) $\quad 79$

Gyõrffy, St., and A. Kohán, Tolerance of Contact Lenses 1

Halberg, J. P., et J. P. Doret, Tuberculose de la chorö̈de dans un cas de tuber-

culose miliaire trailé par la streptomycine ...... 278

Discussion: Semadeni, Bietti, Dufour, R. Bruckner. Hegner, H., und A. Bangerter, Augen-

Dauerbäder ..... 244

Diskussion: R. Bruckner.

Huber, A., Die Wirkung der Calcium-Therapie auf das Auge . . . $\quad .235$

Jablonski, W., vide Frischer, M.

Jacot, P.: Allons-nous oublier le P501? 290

Jonkers, G. II., On the Relation Between the Results of Dark-Adaptation Mea

surements .350

Kaelín-Sulzer, M., Neurinom im Foramen jugulare 255

Kahán, A., vide Györffy, St.

Klainguti, R., Herdförmige Irisatrophie bei Morbus Behçєt .

Diskussion: Knapp, Goldmann.

- $\quad$ Über Kaslanienslachelverle $1 /$ sungen des Auges ..... 247

Knecht, F., Untersuchungen über Witlerungseinflüsse auf die nicht akuten

Glaukomformen 212

Knüsel, 0., Zwei Fäl·1·e von Cataracta electrica

Diskussion: Blum.

- Über Kontaktschalen 225

Kurz, 0., Über Papillitis arteriosclerotica 281

Loebel, M., vide Fríscher, M.

Moeschlin-Sandoz, Y'., Seniler Macula-Pseudotumor mit multiplen Herden . 272 Moginier-

Forel, A., Le traitement des névrites optiques par Гacide nícolinique 304

Discussion: Girordet.

Morsier, G. de, et C. Balavoine, Spasmes de la convergence .... 248

Nérieth, L., Surgical Treatment of Ectropion Caused by Blepharoadenitis . 162

Neuenschwander, M., Placentatherapie der Retinitis pigmentosa . . 262 
Diskussion: Waardenburg, Bíetti. Pajtás, J., Blepharoconjunctivitis allergica après diocaïne .... 144 Pascal, J. I., A New Approach to the Theory of Retinoscopy ... 10

- $\quad$ Role of Corneal Variability in Contact Lenses 318

Radnót, M., Scleritis progressiva posterior 167

Rehsteiner, K., Ein weiterer schweizerischer Stammbaum von dominant ver-

erbter Retinitis pigmentosa 260

Diskussion: Waardenburg, Huber. Richner, H., Die Verwendung des ionisierten und ozonisierlen

Wasserdampf€S

(«lonozon») in der Augenheilkunde

Diskussion: Jacot.

Rintelen, F., Zur Therapie des Blepharospasmus mit Parpanit . 217

Diskussion: A. Bruckner, Knapp, Schlußwort: Rintelen. Sie-Boen-Lian, Epidemic KeratoConjunctivitis in Batavia during 1946 to 1947 . 86 Sobhy Bey, A/., Ma méthode actuelle pour $\Gamma$ Extraction de la cataracte senile.

Mon experience dura $\pi \mathrm{t}$ les 40 dernières années .... , 38

Speiser, S., Über die Beeinflussung der kritischen Flimmerfrequenz durch

verschieden $\Lambda$ veite Pupillen 357

Streiff, E.-B., La symptomatologie oculaire du syndrome cervical postérieur 292 Verrey, F., Terrain, conditions et facteurs de Гinflammation endoculaire . 204 Vönöczky, J., vide Boros, B. Vrabec, Fr., Contribution à Гétude de la genèse des kystes libres intra-oculaires 129

Wilmer, R., Über ein Haemangioma cavernosum der Chorioidea . . $\quad .285$

- $\quad$ Über eine Periphlebitis retinae vom Typus Boeck ..... 288

GESELLSCHAFTSBERICHTE

SOCIETY TRANSACTIONS - SOCIÉTÉS

Vereinigung der Basler Augenärzte. 28. Januar 1948 ..... 107 Groupe ophtalmologique du

Léman, Reunion du 8 dec. 1946 à Lausanne . 124 Schweizerische Ophthalmologische

Gesellschaft, 40. Generalversammlung in Locarno am 4./5. Oktober 1947. - Société Suisse

d'Ophtalmologie, 40e Assemblée générale à Locarno, 4 et 5 octobre 1947 .... 193

NECROLOGIA

In Memoriam Ernst Fleischer, Basel Prof. Dr. August Siegrist $\dagger$

128371

BUCHBESPRECHUNGEN - BOOKS REVIEW

LIVRES NOUVEAUX

$64,128,192.311,372$ 